\title{
5G: THE NEXT GENERATION MOBILE NETWORK, THE ROAD TO A SUPER CONNECTED WORLD.
}

\author{
G.Sriranjani Iyer ${ }^{1}$
}

Abstract : With the ever increasing demand of mobile users, no matter how fast cellular networks are evolving, they don't seem capable of coping up with either the network that runs them or the content that can be delivered through them. The planning for future cellular radio networks is struggling with the challenges of high capacity demand but for low cost. Mobile device users have a voracious appetite for cellular bandwidth, driving app. usage and faster connection speeds. The current cellular technologies such as 3G were inadequate to meet the demands. So in addition to developing technologies like 4G and LTE, approaches such as reducing cell sizes are needed to increase network user capacity. The paper focuses on the 5th Generation networks based on the LTE Advanced Pro. Technology thereby aiming to provide high reliability, robust security and widespread coverage.

Keywords : 1G, 2G, 3G, 4G, 5G, LTE Advanced Pro, 3D Beamforming , NOMA.

\section{INTRODUCTION}

Cellular communication has shown significant improvement over the years in the manner in which people communicate with each other. With the ever increasing demand by the mobile users for better coverage and capacity, the wireless communication technology has seen an evolution starting from 1G in 1980's to the current 4G LTE. In order to provide faster, reliable, more responsive and ubiquitous coverage, The Mobile and Wireless Communication (METIS) Enablers for the Twenty Twenty Information Science project of European Union started research work of 5G at the end of 2012. 5G stands for $5^{\text {th }}$ generation is the next generation of mobile connectivity technologies that supports mobile broadband as well as networking of billions of devices. It has a flexible infrastructure capable of handling ever increasing demand for mobile data and providing connectivity for future technologies such as Internet of Things (IoT), the future of mobile communication.

\section{COMPARITIVE STUDY OF 1G TO 5G SYSTEMS}

1G : The first generation systems called Advanced Mobile Phone Systems (AMPS) evolved in the early 1980's and provided flawless mobile connectivity introducing mobile voice services.

$2 \mathrm{G}$ : The second generation systems are still the most widespread technology in the world. It is a digital wireless technology that increased voice capacity. They are either TDMA or CDMA based. TDMA is used in Global System for Mobile Communication or GSM that operates in the $850 \mathrm{Mhz}$ and 1900Mhz bands in the US and 900Mhz and 1.8Mhz bands in the rest of the world.

3G : The third generation systems optimized mobile for data, enabling mobile broadband services, thus representing the convergence of $2 \mathrm{G}$ wireless systems into a single global system. It is also called Universal Mobile Telecommunications Standard (UMTS). One of the major advantage of UMTS is its global roaming capabilities, they use CDMA techniques and include UMTS (W-CDMA), CDMA 2000, TD-SCDMA.

4G : 4G LTE (Long Term Evolution) or fourth generation systems delivers more capacity for faster and better mobile broadband experiences. It is described as MAGIC :mobile multimedia, anytime anywhere, global mobility support, integrated wireless solution and customized personal service. In this system the carriers use orthogonal frequency division multiplexing (OFDM). According to ITU a 4G network requires a mobile device to be able to exchange data at $100 \mathrm{Mbit} / \mathrm{s}$.

5G : 5G mobile telecommunication standards for $5^{\text {th }}$ generation systems comprise of packet switched wireless systems using orthogonal frequency division multiplexing. The standard is expected to be introduced in 2020's and provides users with 1000 times greater bandwidth as well as 100 times larger data rate to cover huge applications for future mobile stations. The specifications are capable of providing Wireless World Wide Web Applications (WWWW).

\section{PROPOSED TECHNOLOGY FOR 5G}

The technology proposed for $5 \mathrm{G}$ focuses on three major aspects:

1. LTE Advanced Pro Technology,

2. 3D Beamforming,

\footnotetext{
${ }^{1}$ B.E(Hons.), A.M.I.E.T.E, M.Tech Scholar, Department of Electronics and Communication at NRI Institute of Research and Technology Bhopal, India.
} 


\section{Non Orthogonal Multiple Access (NOMA)}

\subsection{LTE Advanced Pro Technology}

In this technique the following points are taken into account:

- Aggregation of carriers: upto 32 carriers including aggregation of licensed spectrum with vast amount of unlicensed spectrum,

- Aggregation across different cells to enhance heterogeneous networks,

- Beyond aggregation the latency is also significantly reduced by evolving LTE frame structure and more responsive connections,

- Increase in flexibility so that operators can dynamically fine tune the traffic conditions,

- Use of many more antenna's at the base station exploiting 3D Beamforming to increase the capacity and coverage.

\subsection{Beamforming :}

- It is a new technology which is used in 5G wireless networks to increase both capacity and coverage,

- 3D Beamforming adapts the radiation beam pattern in both elevation and azimuth planes to provide more degrees of freedom to supporting users,

- To achieve the directionality of the array when transmitting, the phase and relative amplitude of the signal at each transmitter can be controlled to create a pattern of constructive and destructive interference in the wavefront and when receiving information from different sensors is combined in such a way to improve the reception performance of the receiving antenna array,

- To achieve this goal some spatial processing techniques is used in smart antenna's; these are referred to as Beamforming techniques because they can form the array beam pattern to meet the requirements dictated by wireless system. Thus 3D Beamforming:

$>$ Boosts capacity in the urban jungle by increasing SNR and by supporting higher user densities,

$>$ By using active antenna arrays at the base station following things are achievable

i. Adaptive UE specific 3D MIMO/Beamforming in both vertical and horizontal directions (MU-MIMO),

ii. Vertical cell splitting (sectorization),

iii. Practical Cell Shaping.

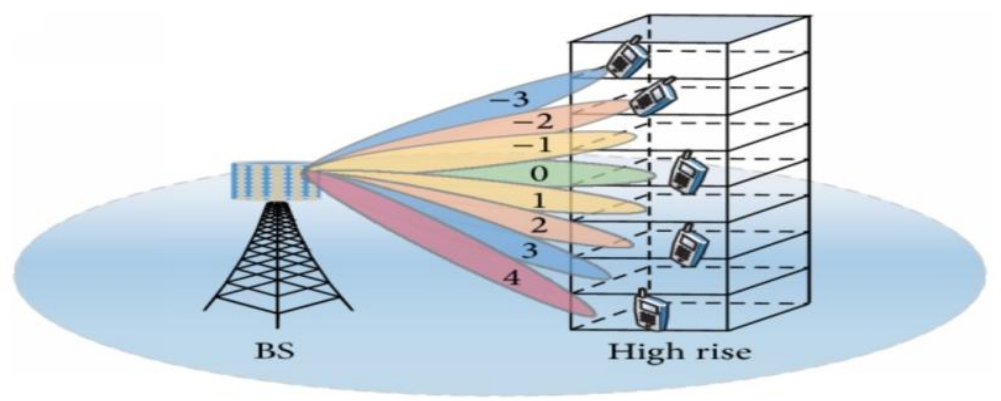

Fig1.3D Beamforming

\section{NON ORTHOGONAL MULTIPLE ACCESS}

- In order to co-ordinate and guarantee services for multiple users some form of multiple access technique is required like $1 \mathrm{G}$ used FDMA, 2G used TDMA, 3G used CDMA and 4G LTE used OFDMA,

- The number of users that can be supported by orthogonal multiple access (OMA) is however limited by the number of available orthogonal dimensions and in practice orthogonality is often lost owing to the effects such as frequency selectivity of channel, phase noise and frequency offsets,

- The above shortcomings of OMA can be overcome by NOMA which is an intercell multiuser multiplexing scheme that proposes the use of an additional domain that is the power domain which has not been exploited in $2 \mathrm{G}, 3 \mathrm{G}$ and $4 \mathrm{G}$ systems,

- At the transmitter side, the user's data is multiplexed on the power domain which means less power allocated for user equipment located near the base station and more power allocation for far cell users,

- NOMA supports simultaneous connections which is suitable to address the challenges related to massive user connectivity, 
- User multiplexing on NOMA is performed without relying on the knowledge of transmitter for the instantaneous channel state information of each user,

- Successive interference cancellation is employed at near users which also has adequate channel to decode the far users data first and cancel out interference,

- According to studies NOMA enhances the reception capacity and cell edge user throughput performance,

- Also NOMA uses superposition coding for downlink transmission where all the users data is combined together and then transmitted,

- Thus, the use of NOMA technique allows the 5G technology to have high spectral efficiency and massive user connectivity.

\section{ARCHITECTURE OF 5G}

- The 5G network architecture consists of all Radio Access Network's (RAN's), aggregator, IP network, nano core etc.

- The 5G system model is exclusively IP based model designed for wireless and mobile networks.

- The 5G network uses flat IP concept that enables different RAN's use the same single nanocore for communication.

- The system comprises of a main user terminal and then a number of independent and autonomous radio access technologies.

- $\quad$ Each of the radio technologies are considered as the IP link for the outside internet world.

- RAN's supported by 5G architecture are GSM, GPRS/EDGE, UMTS, LTE, LTE Advanced, WiMax, WiFi, CDMA 2000, EV-DO, CDMA One, IS-95 etc.

- Flat IP architecture identifies devices using symbolic names unlike hierarchical architecture wherein normal IP addresses are used.

- This architecture reduces number of network elements in data path and hence reduces cost to a greater extent. It also minimizes latency.

- $5 \mathrm{G}$ aggregator aggregates all the RAN traffic and routes it to gateway. 5G aggregator is located at BSC/RNC place.

- 5G mobile terminal houses different radio interfaces for each RAT in order to provide support for all spectrum access and wireless technologies.

- The 5G master core is convergence point for the other technologies which have their own impact on existing wireless network.

- It's design facilitates master core to get operated into parallel multimode including all IP network mode and 5G network mode.

- In this mode it controls all the network technologies of RAN and different access networks. Since the technology is compatible and manages all the new deployments based on $5 \mathrm{G}$, it is more efficient, less complicated and more powerful.

- Any service mode can be opened under 5G new deployment mode as World Combination Service Mode (WCSM).

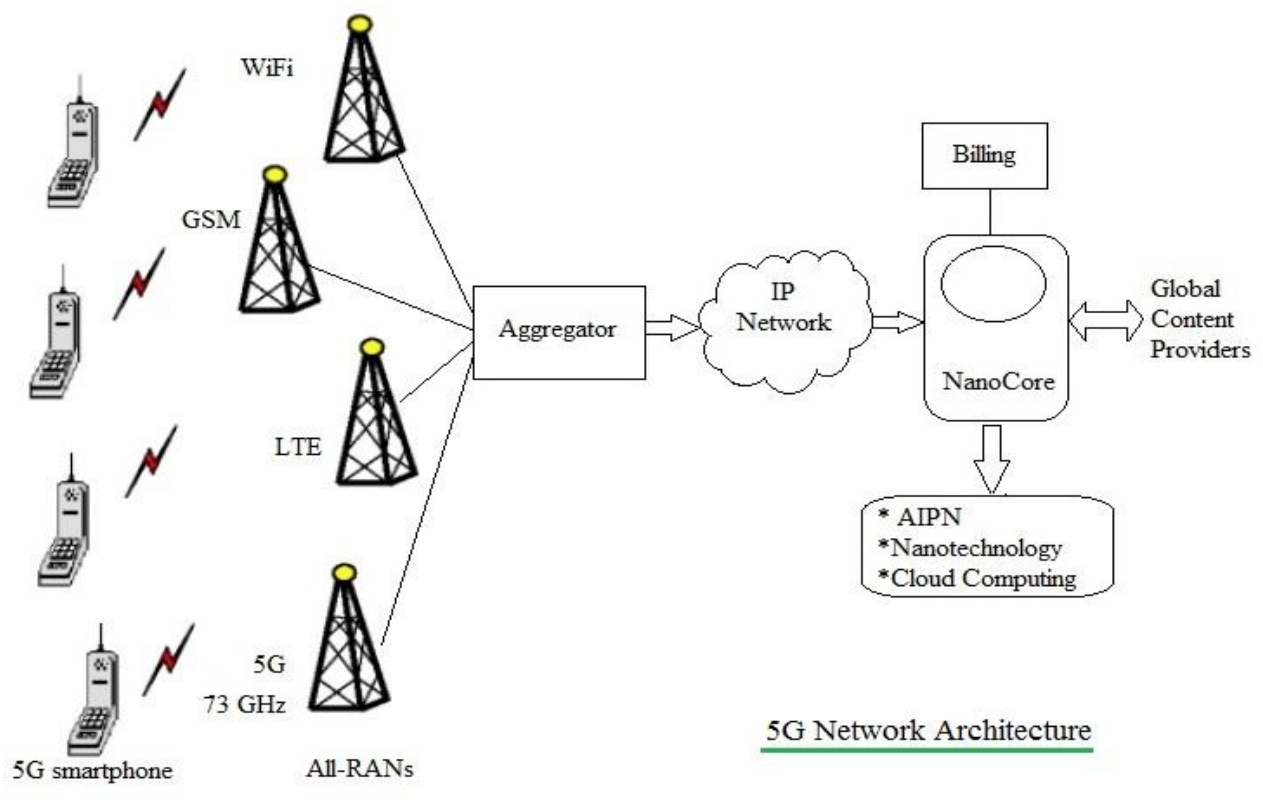

Fig 2. 5G Network Architecture 


\section{FEATURES OF 5G}

- $5 \mathrm{G}$ provides users with 1000 times greater bandwidth as well as 100 times larger data rate to cover huge applications thereby providing networks that are fast and reliable.

- $\quad$ End to End (E2E) quality required by applications and users will be far more diversified in 5G.

- It will provide for wearable devices with artificial intelligence (AI).

- The fifth generation mobile network will use Internet Protocol version 6 (IPv6) wherein the IP address is assigned according to the location and the network to which it is connected.

- $5 \mathrm{G}$ technology is expected to have a speed of 1Gigabit per sec and can support nearly 7000 connections.

- Smart Radio: In order to share the same spectrum efficiently during a wireless transmission scheme, the system will adaptively find unused spectrum. This dynamic radio resource management will be achieved in a distributed manner and rely on software defined transmission.

- Pervasive networks providing ubiquitous coverage: The user can simultaneously be connected to several wireless access technologies and seamlessly move between them.

- High altitude stratospheric platform station (HAPS) systems: The radio interface of 5G communication system is suggested in a Korean research and development program to be based on Beam Division Multiple Access (BDMA) and group is co-operative relay techniques.

\section{ADVANTAGES AND DISADVANTAGES OF 5G}

\subsection{Advantages}

- With 5G technology data rates of about $10 \mathrm{Gbps}$ or higher can be achieved thereby providing better user experience as download and upload speeds are higher.

- It also provides high resolution for cell phone users and bidirectional large bandwidth shaping.

- The advanced billing interfaces of 5G technology makes it more attractive and effective.

- Latency of less than 1millisecond can be achieved in 5G $\mathrm{mm}$ wave. This leads to immediate connection establishment and release with $5 \mathrm{G}$ network by $5 \mathrm{G}$ smartphones, thus reducing traffic load on $5 \mathrm{G}$ base stations.

- With large broadcasting of data at Gigabit rates it can support almost 65000 connections.

- Higher bandwidth can be used with the help of carrier aggregation feature.

- With remote management offered by $5 \mathrm{G}$ technology, a user can get better and faster solution.

- Antenna size is smaller at higher frequencies. This leads to use of massive MIMO concept to achieve higher data rates.

- $\quad 3 D$ Beamforming is employed to overcome path loss at higher frequencies.

- Handoff is smooth due to improved 5G network architecture.

\subsection{Disadvantages}

- The technology is still in developmental stage and hence it will take some time before it is fully operational without any glitches.

- The privacy and security issues are yet to be solved.

- The infrastructure development of 5G technology is very expensive.

- Many of the old devices may not be compatible with 5G, hence all of them needs to be replaced with new one.

- The speed this technology claims seems difficult to be achieved in future, it might be because of lack of technological support in most parts of the world.

\section{REFERENCES}

[1] Guangyi Liu and Dajje Jiang, March 2016, Chinese Journal of Engineering, 5G: Vision and Requirements of mobile communication systems towards year 2020

[2] Sapana Singh and Pratap Singh, August 2012, International Journal of Scientific Research Engineering and Technology, Key Concepts and Network architecture for $5 \mathrm{G}$ mobile technology.

[3] D. Hemalatha, D. Ramakrishna Reddy, K. Sudha, Azmath Mubeen, 2014,Vol 5/6, International Journal of Computer Science and Information Technologies, A study on $5^{\text {th }}$ Generation Mobile Technology - Future Network Service.

[4] Mr. Arunabha Debnath, Mr. Abhinandan, Vol. 1, Issue 4, International Journal on Recent and Innovation Trends in Computing and Communication, Review on Next Generation Technologies of wireless communication.

[5] Andy Sutton, Rahim Tafazolli, Vol. 9, The Journal, 5G, The Future of Mobile Communications

[6] www.Qualcomm.com The Evolution of Mobile Technologies 1G-2G-3G-4G LTE.

[7] B Hrishikesh Vekatraman, Ramona Trestian, 5G Radio Access Networks, Centralised RAN, Virtualization of small cells.

[8] Ramona Sahoo, Gagan Sahoo, Computer Science with C++.

[9] Wikipedia the free Encyclopedia.

[10] Vincent W.S Wong, Robert Schober, Derrick Wing Kwan NG, Li-Chun Wang, Key Technologies for 5G Wireless Systems. 